\title{
Orthodontic Bonding to Unconventional Surfaces - A Review
}

\author{
Nanda Kishore P. ${ }^{1}$, Vikram Shetty², Adeeba Khanum³ ${ }^{3}$ Keertana Rao ${ }^{4}$ \\ ${ }^{1}$ Department of Orthodontics and Dentofacial Orthopaedics, A.B. Shetty Memorial Institute of Dental Sciences, \\ Mangalore, Karnataka, India. ${ }^{2}$ Department of Oral and Maxillofacial Surgery A.B. Shetty Memorial Institute of Dental \\ Sciences, Mangalore, Karnataka, India. ${ }^{3}$ Department of Cleft and Craniofacial Orthodontics A.B. Shetty Memorial \\ Institute of Dental Sciences, Mangalore, Karnataka, India. ${ }^{4}$ A.B. Shetty Memorial Institute of Dental Sciences, \\ Mangalore, Karnataka, India.
}

\section{ABSTRACT}

\section{BACKGROUND}

The introduction of the acid etching bonding technique by Buonocore in 1955 has led to dramatic changes in dentistry. A decade later, Newman used this concept for the use of orthodontic adhesive to bond orthodontic brackets directly onto teeth. Over the past 40 years, continuous developments in dental material sciences and bioengineering resulted in modern orthodontic bonding systems and fixed appliances. Ceramics, resin composites, dental amalgams and acrylic resins are commonly used materials in restorative dentistry and prosthodontics. Most dental ceramic and metal ceramic crowns, bridges and veneers presently are made from different feldspathic porcelains containing $10 \%$ to $20 \%$ aluminium oxide. However, such restorations can also be made from high-aluminium porcelains and glass ceramics. Adhesive-porcelain interface failure is the predominant mode of bond failure in all groups. Conventional acid etching is ineffective in the preparation of porcelain surfaces for mechanical retention of brackets. The most reliable procedure for bonding orthodontic brackets to porcelain surfaces is through the surface treatment combinations of three methods: sandblasting, $9.6 \%$ hydrofluoric acid treatment and silane coupling agent application. As more adults seek orthodontic treatment, we are often faced with the challenge of bonding to restorative materials other than porcelain. The goal throughout orthodontic treatment is to maintain the appliances securely attached to their original position until the end of treatment. However, the appliances should be easily removed without causing any permanent damage to tooth structure or permanent restorations, once the treatment has been concluded. This article reviews the materials and techniques used for bonding to surfaces of restorative materials, termed non-conventional surfaces in contrast to the conventional bonding to tooth enamel. Though this requires following a detailed procedure and using both safe and effective bonding materials, the result should be a reliable bond that does not compromise the integrity of the restorative surface.

\section{KEY WORDS}

Orthodontics, Adhesives, Composite Resins, Dental Enamel
Corresponding Author: Dr. Adeeba Khanum, Fellow,

Cleft and Craniofacial Orthodontics, A.B. Shetty Memorial Institute of Dental Sciences, Mangalore-575018,

Karnataka, India.

E-mail:dradeebaortho@gmail.com

DOI: $10.14260 / \mathrm{jemds} / 2020 / 616$

How to Cite This Article:

Kishore NP, Shetty V, Khanum A, et al. Orthodontic bonding to unconventional surfaces-a review. J Evolution Med Dent Sci 2020;9(38):2829-2832, DOI: $10.14260 /$ jemds/2020/616

Submission 04-02-2020,

Peer Review 20-03-2020,

Acceptance 27-03-2020,

Published 21-09-2020.

Copyright (C) 2020 JEMDS. This is an open access article distributed under Creative Commons Attribution License [Attribution 4.0 International (CC BY 4.0)] 


\section{BACKGROUND}

Over the past 30 - 40 years, a major technical improvement in orthodontic therapy has come to the forefront, in the process of bonding orthodontic appliances to the tooth enamel. Bonding of attachments, eliminating the need for bands, was a dream for many years before rather abruptly becoming a routine clinical procedure in the 1980s. Bonding is based on the mechanical locks formed on the base of orthodontic attachment and the chemical/mechanical locking of an adhesive to the irregularities on the enamel surface.[1]

\section{Advantage of Bonding over Banding}

- Aesthetically superior.

- Reduced patient discomfort.

- Arch length maintained.

- Brackets can be positioned more precisely.

- Hygienic.

- Partially erupted teeth can be controlled.

- Interproximal areas can be accessed for composite buildup.

- Risk of caries under the loose bands is eliminated.

- Artificial tooth and fixed bridgework surfaces, allow for bonding attachments.

- When esthetics is a priority, invisible braces or lingual brackets may be used.

- No requirement of closing band spaces at the end of treatment.

- Ease of application for clinician.

- The cost can be further reduced by recycling the brackets.

\section{Disadvantages}

- Weaker attachment.

- Though the access for cleaning is better, it doesn't guarantee improved gingival condition and better oral hygiene.

- Protection against interproximal caries of well contoured bands is absent.

- When lingual auxiliaries or headgears are required, bonding is generally not indicated.

- More preparation is required for rebonding.

- Debonding is more time consuming.

\section{Evolution of Bonding in Orthodontics}

In 1970, the first bonding adhesive methyl methacrylate/poly methyl methacrylate with plastic brackets was introduced. Thereafter, two paste chemical cure system (1972), ultraviolet light cure system (1974), no mix chemical cure adhesive (1975), brazed foil mesh metal brackets (1979), visible light cure adhesive (1983), multisurface hydrophilic primer (1985), colour change adhesive (1986), dual cure glass ionomer cement (1995), prepasted brackets (1996), new generation hydrophilic primers (1998), self-etching primers (2000), nonporous enamel protective sealants (2003), light cure colour change adhesives (2004), all surface hydrophilic primers (2014) have been launched..[2]

The extent of orthodontics has progressed in the course of recent decades to incorporate increasingly adult patients and it is normal that a considerable lot of these individuals will have restorations of porcelain, amalgam, casting alloys, resin composites or acrylic resins placed on their teeth. The objective during orthodontic treatment is that the appliances remain safely joined to their original position until the culmination of treatment. [3]

Once the treatment is completed, the appliances should be handily removed without causing any perpetual harm to the tooth structure or permanent restorations. ${ }^{[1]}$

\section{Bonding to Porcelain}

Some adult patients have porcelain surfaces on certain teeth and may require orthodontic brackets and retainer wires to be fitted. In these cases, conventional acid etching is ineffectual. In 1986 Wood et al. demonstrated that roughening the porcelain surface, including a porcelain primer, and utilizing a highly filled adhesive resin when bonding to glazed porcelain added increasingly to the bond strength. ${ }^{[4]}$

\section{METHODS OF SURFACE PREPARATION [5]}

1. Mechanical Roughening with Stones and Diamonds Bond strength can be increased by roughening the porcelain surface with diamonds or stones. Although, sufficient retention for the entire orthodontic treatment period cannot be obtained. Micro cracks may be produced on the ceramic surface, due to grinding, which can cause fracture of the restoration.

\section{Micro-Etching}

50 um or coarser aluminium oxide powder connected to a compressed air source in the operatory is used via small intra oral sandblasters with a contra-angle nozzle. It is accomplished with rubber dam isolation and heavy duty suction for collection of ejected powder. Silicon carbide could be used in patients allergic to aluminium oxide, as a substitute powder.

\section{Chemical Coupling}

Adhesion of resin composites can be enhanced by application of silane. A coupling agent that provides reactive sites for inorganic and organic components is gamma-methacryloxypropyltrimethoxysilane. This silane comprises the silanol groups which form a siloxane (si-osi) bond by interacting with silanols on the ceramic surface. It also consists of methacrylate groups which form covalent bonds with the polymer matrix of resin composite.

\section{Chemical Roughening}

Etching of ceramic surface with hydrofluoric acid was presented in 1980s. Hydrofluoric acid (HF) is very potent and requires extensive care in handling and segregation with a rubber dam. HF $9.6 \%$ in gel form is the most frequently used concentration. It is applied on the ceramic surface for 2 to 4 mins. $4 \%$ acidulated phosphate fluoride consisting of $1.43 \% \mathrm{HF}$ in gel form 
for 2 minutes can also be utilized. For areas of ceramic where isolation is difficult, $1.23 \%$ acidulated phosphate fluoride gel can be used for 10 minutes. HF etching without roughening of ceramic surface produces adequate micro porosities to achieve effective bonding.

Schmage et al[6] performed a study in which they measured the effect of various surface- methods on the bond strength of metal brackets to ceramic surfaces. They used shear bond strength and surface roughness tests to analyse the impact of four different methods of surface conditioning: fine diamond bur, sandblasting, $5 \% \mathrm{HF}$, and silica coating to bind metal brackets to ceramic surfaces of feldspathic porcelain. Sandblasting and silicatization with silane or HF without silane obtained the highest bond strength values. The highest surface roughness was shown by diamond roughening and sandblasting; they can damage the ceramic surface.

Zachrisson et al concluded that HF produces wide penetrating patterns (in depth). But diamonds roughening and micro etching only cause surface peeling. [5]

Gursimrit et al used a systematic analysis to decide which materials and technique/protocol pose the highest rate of success on porcelain surfaces when bonding brackets. The best protocol listed in this analysis is HF etched for 1 minute at $9.6 \%$, rinsed for 30 seconds, and then air-dried. A silane application should be followed after etching with the HF. Taking into account the adverse effects of etching with HF, they propose mechanical roughening with sandblasting followed by a silane application. ${ }^{[7]}$

\section{Bonding to Amalgam}

A small amalgam filling with surrounding sound enamel or a large amalgam restoration or amalgam can be bonded with the following improved methods only

1. Metal surface modification (sandblasting, diamond bur roughening)

2. Use of intermediate resins to improve bond strengths (e.g., All-bond 2, enhance, and metal primer)

3. New adhesive resins that chemically bind to non-precious and precious metals (e.g. 4-[4-META] resins and 10-MDP bis-GMA resins)

Sandblast the amalgam alloy with $50 \mu \mathrm{m}$ aluminium oxide for 3 seconds for a small amalgam filling with surrounding sound enamel. Prepare the surrounding enamel for 15 seconds with $37 \%$ phosphoric acid. Apply sealant and bond with composite resin, and ensure the bonded attachment is not in occlusion with antagonists. Apply a uniform coat of metal primers instead of phosphoric acid, for a large amalgam restoration or amalgam only and wait 30 seconds. ${ }^{[8]}$

\section{Bonding to Gold}

Unlike bonding to porcelain and amalgam, the orthodontic practitioners don't seem to have outstanding bonding to gold crowns. Different new technologies have been documented to improve bonding to gold in laboratory settings, including sandblasting, electrolytic tin-plating or gallium-tin solution plating.
Tamer et al [9] conducted a study to compare the effect of various surface treatments on bonding to gold alloy. Cast gold alloy was subjected to one of the three surface treatments-

1. Roughening with diamond bur.

2. Aluminum oxide sandblasting.

3. Sandblasting and tin electroplating.

After application of an intermediate resin they used Concise or Superbond. They concluded that sandblasting created stronger gold alloy bonds compared to roughening with diamond bur. Tin-plating strengthened the orthodontic bond to sandblasted alloy gold, just slightly. Concise specimens typically came loose in the gold alloy/adhesive interface with all the adhesive remaining on the underside of the bracket. The fractures occurred either in the adhesive interface or in the adhesive/bracket interface with Superbond.

\section{Bonding to Composite Restoratives}

The bond strength obtained by adding new composite to existing composite is considerably less than the material's cohesive strength. Nevertheless, in most cases, brackets bonded to a new, roughened surface of old composite restorations tend to be clinically effective. It is also likely to be beneficial to use an intermediate primer.[10]

\section{Bonding to Fluorosed Teeth}

Due to regular bracket failure at the damaged enamel interface, bonding brackets to fluorosed teeth remains a notable clinical problem. Fluorosed teeth have increased subsurface enamel porosity or hypo-mineralization, irregular patterns of prism, cracks and fissures, and well mineralized enamel surface layer. Such anomalies lead to a marked reduction in microhardness, which adversely affects the mechanical locking of an adhesive to the surface of the enamel.

Severely fluorotic enamel is vulnerable to fracture because highest susceptibility to mechanical stress is in the well mineralized region. Enamel with a high content of fluoride is more etching-resistant and results in less irregularities. It has been proposed that this can result in weaker resin bond and, ultimately, poor orthodontic bracket retention.

Duan et al recommended grinding the surface layer of enamel in fluorosed teeth and then polishing before etching. When the fragile outer layer of enamel is removed, the micro hardness of the enamel is improved, so that the retentive tags within the enamel can endure greater mechanical force. Failures are further reduced by augmenting the adhesive surface with a light cured veneer because bond strength is directly proportional to the surface area.[2]

James et al proposed that the use of adhesion promoters for extremely fluorosed human teeth would eliminate the need for micro-abrasion.[11] A study by Al Sugair et al reported that the etching of slightly fluorosed teeth with white lines or distinct opaque areas would be the same as for non-fluorosed teeth in clinical practice. The etching time of moderately fluorosed teeth with nearly opaque enamel will, however, be at least doubled. In the case of extreme fluorosed teeth with pitted or focal loss of surface enamel, the etching time (at least 90 seconds) should be increased to clear both the hypermineralized surface layer and the organic network as well as to create typical etching patterns.[12] 


\section{CONCLUSIONS}

Bonding brackets has transformed the art of orthodontics, and in a relatively short period it has become a standard clinical procedure. If the appropriate technique is used, bonding may be effective in achieving the optimum bond strength. Although no adhesive is foolproof, if handled correctly, any system can achieve the desired results.

Financial or Other Competing Interests: None.

\section{REFERENCES}

[1] Proffit WR, Fields Jr HW, Sarver DM. Contemporary Orthodontics. $4^{\text {th }}$ edn. Elsevier Health Sciences 2006.

[2] Gange P. The evolution of bonding in orthodontics. Am J Orthod Dentofac Orthop 2015;147(Suppl 4):S56-S63.

[3] Brantley WA, Eliades T. Orthodontic materials: scientific and clinical aspects. Stuttgart: Thieme 2001: p. 310.

[4] Wood DP, Jordan RE, Way DC, et al. Bonding to porcelain and gold. Am J Orthod 1986;89(3):194-205.
[5] Zachrisson YO, Zachrisson BU, Büyükyilmaz T. Surface preparation for orthodontic bonding to porcelain. Am J Orthod Dentofac Orthop 1996;109(4):420-30.

[6] Schmage P, Nergiz I, Herrmann W, et al. Influence of various surface-conditioning methods on the bond strength of metal brackets to ceramic surfaces. Am J Orthod Dentofac Orthop 2003;123(5):540-6.

[7] Bach GKG, Torrealba Y, Lagravère MO. Orthodontic bonding to porcelain: a systematic review. Angle Orthod 2014;84(3):555-60.

[8] Zachrisson BU, Büyükyilmaz T, Zachrisson YO. Improving orthodontic bonding to silver amalgam. Angle Orthod 1995;65(1):35-42.

[9] Büyükyilmaz T, Zachrisson YO, Zachrisson BU. Improving orthodontic bonding to gold alloy. Am J Orthod Dentofac Orthop 1995;108(5):510-8.

[10] Graber TM, Vanarsdall RL. Orthodontics, current principles and techniques. $3^{\text {rd }}$ edn. CV Mosby Co. 2000;53(9):1689-99. doi:10.1017/CB09781107415324.004

[11] Noble J, Karaiskos NE, Wiltshire WA. In vivo bonding of orthodontic brackets to fluorosed enamel using an adhesion promotor. Angle Orthod 2008;78(2):357-60.

[12] Al-Sugair MH, Akpata ES. Effect of fluorosis on etching of human enamel. J Oral Rehabil 1999;26(6):521-8. 